\title{
Efeitos da Injeção de Cloreto de Cálcio Pós-morte e Tempo de Maturação no Amaciamento e nas Perdas por Cozimento do Músculo Longissimus dorsi de Animais Bos indicus e Bos taurus Selecionados para Ganho de Peso ${ }^{1}$
}

\author{
Aparecida Carla de Moura ${ }^{2}$, Albino Luchiari Filho ${ }^{3}$, Romeu Fernandes Nardon ${ }^{4}$, Alexander \\ George Razook
}

\begin{abstract}
RESUMO - O objetivo deste estudo foi avaliar o efeito da injeção pós-morte de cloreto de cálcio $\left(\mathrm{CaCl}_{2}\right)$ e o tempo de maturação no amaciamento e nas perdas por cozimento do músculo longissimus dorsi de animais Bos indicus e Bos taurus selecionados para ganho de peso. Foram usados 64 machos inteiros (16 Caracu, 16 Guzerá, 16 Nelore Controle e 16 Nelore Seleção). Vinte quatro horas após o abate, foi retirada uma amostra do músculo Longissiumus dorsi (contra-filé) entre a 6⿳亠 a e 9a vértebras lombares e dividida em nove subamostras. Em cada grupo de três subamostras escolhidas ao acaso, foi injetada, na quantia correspondente a $10 \%$ do seu peso, uma das seguintes soluções: a) água (controle), b) $200 \mathrm{mM} \mathrm{de} \mathrm{CaCl}_{2}$ e c) $300 \mathrm{mM} \mathrm{de} \mathrm{CaCl}_{2}$. Cada subamostra foi, então, embalada a vácuo, congelada $\left(-2^{\circ} \mathrm{C}\right)$ e maturada por 1,7 ou 14 dias até a realização de testes de força de cisalhamento e perdas por cozimento (evaporação, gotejamento e perdas totais). Foi usado delineamento experimental completamente casualizado com parcelas subdivididas, em que a parcela correspondia à raça e a sub-parcela, à combinação entre três níveis de $\mathrm{CaCl}_{2}$ e três tempos de maturação. A raça influenciou a força de cisalhamento, mas não influiu nas perdas por cozimento A maturação por um período de sete dias reduziu os valores de força

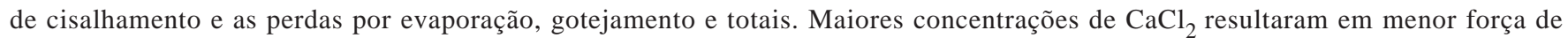
cisalhamento e maiores perdas por evaporação, embora não tenham influenciado as perdas por gotejamento e totais. A concentração de $200 \mathrm{mM} \mathrm{CaCl}_{2}$ apresentou a melhor redução para a força de cisalhamento. A injeção pós-morte de uma solução de $\mathrm{CaCl}_{2}$ aumentou o processo de amaciamento, sem influir nas perdas por cozimento.
\end{abstract}

Palavras-chave: amaciamento da carne, cloreto de cálcio, Bos indicus, Bos taurus, força de cisalhamento, injeção, Longissimus dorsi

\section{Effects of Postmortem Calcium Chloride Injection and Aging Time on Tenderness and Cooking Losses of Longissimus dorsi Muscle From Bos indicus and Bos taurus Animals Selected for Weight Gain}

\begin{abstract}
The objective of this study was to evaluate the effect of postmortem calcium chloride $\left(\mathrm{CaCl}_{2}\right)$ injection and aging time on tenderness and cooking losses of Longissimus dorsi muscle from Bos indicus and Bos taurus animals selected for weight gain. Sixty-four young bulls (16 Caracu, 16 Guzera, 16 Nellore Control and 16 Nellore Selection) were used. Twenty four hours after slaughter a sample from Longissimus dorsi muscle, taken between the $6^{\text {th }}$ and $9^{\text {th }}$ lumbar vertebrae was removed and divided into nine sub-samples. In each sub-samples, randomly selected, an amount correspondent to $10 \%$ of sub-sample weight was injected, with one of the following solutions: a) water (control), b) $200 \mathrm{mM} \mathrm{CaCl}_{2}$ or c) $300 \mathrm{mM} \mathrm{CaCl}_{2}$. Each sub-sample was then vacuum-wrapped, cooled to - $2^{\circ} \mathrm{C}$ and aged for 1, 7 or 14 days until the realization of the shear force and cooking losses (evaporation, drip, and total losses) tests. A completely randomized design with a split-plot arrangement, where breeds corresponded to a whole plots and the combinations among three levels of $\mathrm{CaCl}_{2}$ and three aging times as split-plots, was used. The breed affected the shear force, but did not affected the cooking losses. Higher $\mathrm{CaCl}_{2}$ concentrations resulted on the lowest shear force values and greater evaporation losses although it did not affect either dripping or total losses. The $200 \mathrm{mM} \mathrm{CaCl}_{2}$ concentration showed the best reduction in the shear force. The postmortem injection with $\mathrm{CaCl}_{2}$ hasten the tenderness process without affecting the cooking losses.
\end{abstract}

Key Words: meat tenderness, calcium chloride, Bos indicus, Bos taurus, shear force, injection, Longissimus dorsi

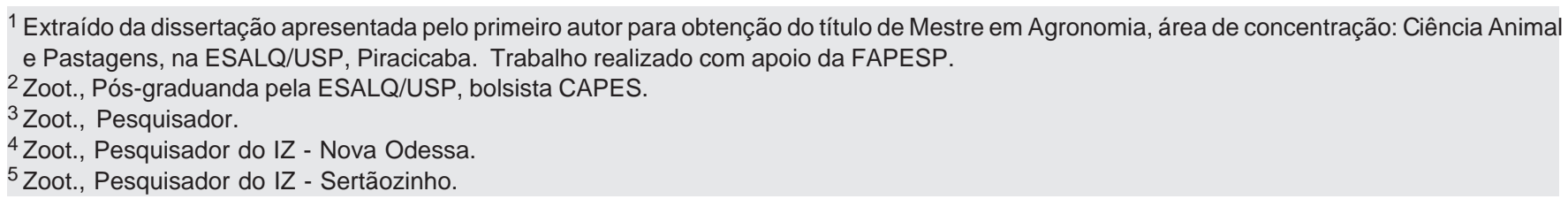




\section{Introdução}

Nos últimos 10 anos, o rebanho bovino brasileiro sofreu inúmeras mudanças, como aumento do rebanho e da produção por abate, uma vez que a população brasileira também cresceu e, por conseguinte, o consumo de carne (devido à estabilização da economia) também aumentou.

Com o novo dinheiro, o Real, valendo mais, o consumidor está se conscientizando de que pode e deve ser exigente na qualidade dos produtos que está adquirindo. Segundo FELÍCIO (1995), a maciez foi eleita em primeiro lugar (64\%) como a característica de qualidade mais importante (mais valorizada).

No Brasil, a carne produzida é, predominantemente, oriunda de animais Bos indicus, considerada menos macia, o que pode ser atribuído à ocorrência do fenômeno de encolhimento pelo frio (FELÍCIO, 1995), em conseqüência de sua menor espessura de gordura, associado ao manejo de resfriamento. Devese considerar também a diferença de atividade das proteases dependentes de cálcio e de seu inibidor, calpastatina, entre estes animais e os animais Bos taurus (WHIPPLE et al., 1990).

Uma vez que a maciez da carne é a característica organoléptica mais importante para o consumidor, uma das soluções para a carne proveniente de animais Bos indicus seria a utilização de métodos tecnológicos para melhorar a sua maciez; entre os mais comuns destacam-se estimulação elétrica, suspensão pélvica, manejo de câmara fria, maturação e utilização de cloreto de cálcio. Todos esses métodos baseiam-se, principalmente, em aumentar certas características de palatabilidade (maciez) por intermédio do aumento do comprimento do sarcômero, da atividade autolítica das enzimas, da liberação de enzimas lisossômicas e da tensão sobre diversos músculos do lombo e da perna, da redução da desnaturação protéica, da perda da força de tensão do componente miofibrilar da célula muscular, devido à desintegração das bandas $\mathrm{Z}$, e da ruptura da fibra muscular.

A utilização de uma solução de cloreto de cálcio (por meio de injeção, marinação ou infusão), em cortes comerciais ou nas carcaças inteiras, é um dos recursos tecnológicos mais recentes para reduzir a variabilidade da maciez (ALARCÓN-ROJOe DRANSFIELD, 1995; LANSDELL et al., 1995; e WHEELER et al., 1993 $\mathrm{a}, \mathrm{b}, \mathrm{c})$. Este processo consiste em fornecer cálcio exógeno para as proteases dependentes de cálcio ( $\mu$-, m-calpaína) e seu inibidor (calpastatina), acelerando o processo de amaciamento por intermédio da ativação de m-calpaína que, em condições normais pós-morte, é pouco ativada, por necessitar de maior concentração de íons cálcio para ser ativada.

Assim, o objetivo deste trabalho foi estudar os efeitos da injeção de cloreto de cálcio sobre a força de cisalhamento da carne de animais Caracu, Guzerá, Nelore Controle e Nelore Seleção, selecionados para ganho de peso.

\section{Material e Métodos}

Foram utilizados 64 machos inteiros, 16 Caracu (C), 16 Guzerá (G), 16 Nelore Controle (NC) e 16 Nelore Seleção (NS), oriundos do projeto de seleção da Estação Experimental de Zootecnia de Sertãozinho. Estes animais permaneceram em confinamento, na Estação Experimental de Zootecnia de Nova Odessa por um período de 177 dias, no qual receberam uma dieta contendo $55 \%$ de concentrado (76,5\% milho, 19,2\% farelo de algodão, 1,62\% uréia, 0,18\% amônia, $1,5 \%$ calcário e $1 \%$ sal mineral) e $45 \%$ de volumoso (silagem de milho e/ou silagem de milho+sorgo). A idade média ao final do confinamento foi de 19,7 meses, com peso vivo ao abate de $473 \mathrm{~kg}$ e rendimento de carcaça de $59,2 \%$.

Após o abate, as carcaças permaneceram por 24 horas em resfriamento (suspensas em câmara fria a $4^{\circ} \mathrm{C}$ ), quando uma amostra do músculo Longissimus dorsi (contra-filé), situado entre a 6 e a 9 $\underline{a}$ vértebras lombares (lado direito), foi retirada para realização deste trabalho.

As soluções de cloreto de cálcio (0, 200 e 300 mM) foram preparadas com água destilada e homogeneizadas no Centro de Biotecnologia da ESALQ em Piracicaba.

O músculo Longissiumus dorsi foi subdividido em nove subpartes (bifes de 2,54 cm de espessura), as quais foram submetidas, ao acaso, aos seguintes tratamentos com injeção de cloreto de cálcio, na base de $10 \%$ do peso da amostra: a) água (controle), b) $200 \mathrm{mM}$ de cloreto de cálcio e c) $300 \mathrm{mM}$ de cloreto de cálcio. As soluções, depois de injetadas por todos os lados nas respectivas amostras, foram embaladas a vácuo e transferidas para uma câmara fria $-2^{\circ} \mathrm{C}$, onde permaneceram por 1,7 e 14 dias, para posterior análise da força de cisalhamento e perdas por cozimento.

A metodologia da força de cisalhamento foi aquela indicada pelo AMERICAN MEAT SCIENCE ASSOCIATION - AMSA (1978), com as seguintes modificações: aos 1ํㅜ $7 \underline{0}$ e $14^{\circ}$ dia após o abate, bifes 
1384 Rev. bras. zootec.

de 2,54 cm de espessura, provenientes do lado direito da carcaça do animal abatido, foram assados em forno a gás, à temperatura de $200^{\circ} \mathrm{C}$, até atingir temperatura interna de $75^{\circ} \mathrm{C}$ (monitoração da temperatura feita com termômetro). Os bifes foram resfriados à temperatura ambiente por 1 hora. Seis cilindros por bife, com 1,27 cm de diâmetro, foram retirados no sentido paralelo a fibra com o auxílio de um razador, e cada um foi cisalhado utilizando-se o Warner Bratzler Shear Force Device, sendo a média entre eles o resultado da força de cisalhamento, expresso em quilogramas. A determinação das perdas por cozimento foi feita pela pesagem das bandejas com as amostras antes e após o cozimento, determinando-se as perdas por gotejamento (peso do líquido restante na bandeja, menos o peso da amostra sem dreno após cozimento, dividido pelo peso da amostra antes do cozimento e multiplicado por 100), as perdas por evaporação (peso da amostra antes do cozimento, menos o peso da amostra após cozimento, dividido pelo peso da amostra antes do cozimento e multiplicado por 100) e as perdas totais (soma entre as perdas por evaporação e perdas por gotejamento).

A análise estatística foi realizada utilizando-se o programa SAS (1990), segundo um delineamento completamente casualizado com parcelas subdivididas ("split-plot"). As quatro raças (Caracu, Guzerá, Nelore Controle e Nelore Seleção) corresponderam às parcelas, enquanto às subparcelas foram designados os tratamentos que correspondem a todas as combinações possíveis entre três concentrações de cloreto de cálcio $(0,200$ e $300 \mathrm{mM})$ e três tempos (1, 7,14 dias) de maturação (em um arranjo 3 x 3 na subparcela). O modelo incluiu os principais efeitos de raça (para cada animal), concentração de cloreto de cálcio e tempo de maturação, as interações entre raça x concentração de cloreto de cálcio e raça $x$ tempo de maturação e a concentração de cloreto de cálcio x tempo de maturação. Os erros incluíram animal (dentro de raça) para a parcela e os termos de interação para a sub-parcela.

\section{Resultados e Discussão}

Influência das interações raça $x$ tempo de maturação, raça x concentração de cloreto de cálcio e tempo de maturação $x$ concentração de cloreto de cálcio

Força de cisalhamento (FC)

Estudando-se a interação raça $x$ tempo de maturação (Tabela 1), observa-se que houve efeito do tempo de maturação sobre as raças $(\mathrm{P}<0,05)$, no qual ocorreu redução significativa na FC do $1^{\circ} \underline{0}$ ao $7^{\circ} \underline{0}$ dia $(0,66-1,41 \mathrm{~kg})$, a qual foi menor $(0,20-0,61 \mathrm{~kg})$ do $7 \underline{\mathrm{o}}$ ao $14 \underline{\mathrm{o}}$ dia, mas de maneira significativa $(\mathrm{P}<0,05)$ e semelhante para todas as raças estudadas. Quanto ao efeito da raça dentro do tempo de maturação no dia 1 de maturação, observa-se que os animais $C$ apresentaram menor FC e os NC, valores de FC semelhantes ou iguais entre os animais de FC intermediário (NS) e alto (G). No entanto, sete dias após maturação, a variação na maciez encontrada foi reduzida, em que os animais $\mathrm{C}$ continuaram com menores valores de $\mathrm{FC}$ e os das demais raças apresentaram altos valores de FC (carne menos macia que o $\mathrm{C}$, mas mais macia que o dia 1 de maturação). Aos 14 dias de maturação, nota-se que os animais C ainda apresentavam carne tão macia quanto os animais NS, porém mais macia que a dos animais G e NC. Com os resultados obtidos, pode-se, assim, verificar que os animais $\mathrm{C}$ apresentaram carne mais macia, em todos os dias de maturação empregados, mostrando o que parece ser influência positiva do sangue Bos taurus sobre a maciez da carne. Animais NC e NS apresentaram FC intermediários, enquanto os animais G, apesar de sofrerem maturação, continuaram a apresentar carne menos macia (maior FC) em relação aos demais animais, mostrando, assim, a possível influência do sangue Bos indicus na dureza da carne. Estes resultados concordam com algumas pesquisas (KOCH et al., 1982; CROUSE et al., 1987 e 1989; e WHIPPLE et al., 1990), em que animais Bos indicus apresentam maior FC. Segundo SHACKELFORD et al. (1995) animais Bos indicus apresentam maior FC para os músculos Longissimus dorsi, Triceps brachii, Supraspinatus, Biceps femoris e Quadriceps femoris.

Comparando-se a interação raça x concentração de cloreto de cálcio, observa-se que houve efeito da concentração de cloreto de cálcio dentro da raça (Tabela 2), em que a FC foi reduzida significativamente, em virtude da aplicação de 200 ou 300 mM de cloreto de cálcio. A aplicação de $300 \mathrm{mM}$ de cloreto de cálcio não foi estatisticamente diferente de $200 \mathrm{mM}$ para as raças $\mathrm{G}, \mathrm{NC}$ e $\mathrm{NS}$, porém reduziu a $\mathrm{FC}$ $(\mathrm{P}<0,05)$ para a raça $\mathrm{C}$ (quanto maior a quantidade aplicada, maior o amaciamento). Os resultados desta pesquisa (amaciamento de 20-32\%) são semelhantes aos mencionados por vários autores (MORGAN et al., 1991; WHEELER et al., 1993a; e LANSDELL et al. 1995), que obtiveram valores na redução da variação 
MOURA et al.

Tabela 1- Médias da força de cisalhamento, das perdas por evaporação, das perdas por gotejamento e das perdas totais em relação à raça, ao tempo de maturação e à interação raça x tempo de maturação

Table 1 - Means of the shear force, evaporation loss, drip loss and total loss by breed, ageing time and breed vs. ageing time interaction

\begin{tabular}{|c|c|c|c|c|}
\hline \multirow{3}{*}{$\begin{array}{l}\text { Raça } \\
\text { Breed }\end{array}$} & \multicolumn{3}{|c|}{ Maturação } & \multirow{3}{*}{$\begin{array}{l}\text { Média } \\
\text { Mean }\end{array}$} \\
\hline & \multicolumn{3}{|c|}{ Ageing time } & \\
\hline & $1 \mathrm{~d}$ & $7 \mathrm{~d}$ & $14 \mathrm{~d}$ & \\
\hline \multicolumn{5}{|c|}{ Força de cisalhamento (Shear force), $\mathrm{kg}$} \\
\hline Caracu & $3,15^{\mathrm{A}, \mathrm{c}}$ & $2,49^{\mathrm{B}, \mathrm{b}}$ & $2,29 \mathrm{C}, \mathrm{b}$ & 2,64 \\
\hline Guzerá & $4,87^{\mathrm{A}, \mathrm{a}}$ & $3,46^{\mathrm{B}, \mathrm{a}}$ & $2,95^{\mathrm{C}, \mathrm{a}}$ & 3,76 \\
\hline \multicolumn{5}{|l|}{ Nelore Controle } \\
\hline Nellore Control & $4,23^{\mathrm{A}, \mathrm{ab}}$ & $3,27^{\mathrm{B}, \mathrm{a}}$ & $2,76^{\mathrm{C}, \mathrm{a}}$ & 3,42 \\
\hline \multicolumn{5}{|l|}{ Nelore Seleção } \\
\hline Nellore Selection & $3,97^{\mathrm{A}, \mathrm{b}}$ & $3,24^{\mathrm{B}, \mathrm{a}}$ & $2,63^{\mathrm{C}, \mathrm{ab}}$ & 3,28 \\
\hline \multirow[t]{2}{*}{ Média(Mean) } & 4,06 & 3,12 & 2,66 & \\
\hline & \multicolumn{3}{|c|}{ Perda por evaporação (Evaporation loss), \% } & \\
\hline Caracu & $15,37^{\mathrm{A}, \mathrm{b}}$ & $15,78^{\mathrm{A}, \mathrm{a}}$ & $15,46^{\mathrm{A}, \mathrm{a}}$ & 15,54 \\
\hline Guzerá & $16,78^{\mathrm{A}, \mathrm{ab}}$ & $15,45^{\mathrm{AB}, \mathrm{a}}$ & $14,57^{\mathrm{B}, \mathrm{a}}$ & 15,60 \\
\hline \multicolumn{5}{|l|}{ Nelore Controle } \\
\hline Nellore Control & $15,71^{\mathrm{A}, \mathrm{ab}}$ & $17,34^{\mathrm{A}, \mathrm{a}}$ & $15,79^{\mathrm{A}, \mathrm{a}}$ & 16,28 \\
\hline \multicolumn{5}{|l|}{ Nelore Seleção } \\
\hline Nellore Selection & $18,34^{\mathrm{A}, \mathrm{a}}$ & $16,99 \mathrm{AB}, \mathrm{a}$ & $15,30^{\mathrm{B}, \mathrm{a}}$ & 16,88 \\
\hline \multirow[t]{2}{*}{ Média (Mean) } & 16,55 & 16,39 & 15,28 & \\
\hline & \multicolumn{4}{|c|}{ Perda por gotejamento (Drip loss), \% } \\
\hline Caracu & $12,72^{\mathrm{A}, \mathrm{a}}$ & $11,03^{\mathrm{B}, \mathrm{a}}$ & $12,12^{\mathrm{A}, \mathrm{a}}$ & 11,96 \\
\hline Guzerá & $11,29^{A, a b}$ & $11,28^{\mathrm{A}, \mathrm{a}}$ & $11,70^{\mathrm{A}, \mathrm{ab}}$ & 11,42 \\
\hline \multicolumn{5}{|l|}{ Nelore Controle } \\
\hline Nellore Control & $11,49^{\mathrm{A}, \mathrm{ab}}$ & $10,53^{\mathrm{A}, \mathrm{a}}$ & $10,86^{\mathrm{A}, \mathrm{b}}$ & 10,96 \\
\hline \multicolumn{5}{|l|}{ Nelore Seleção } \\
\hline Nellore Selection & $10,82^{\mathrm{A}, \mathrm{b}}$ & $9,92^{\mathrm{A}, \mathrm{a}}$ & $11,01^{\mathrm{A}, \mathrm{ab}}$ & 10,59 \\
\hline \multirow[t]{2}{*}{ Média(Mean) } & 11,58 & 10,69 & 11,42 & \\
\hline & & otal (Total lo & & \\
\hline Caracu & $28,09^{\mathrm{A}, \mathrm{a}}$ & $26,81^{\mathrm{A}, \mathrm{a}}$ & $27,58^{\mathrm{A}, \mathrm{a}}$ & 27,49 \\
\hline Guzerá & $28,05^{\mathrm{A}, \mathrm{a}}$ & $26,73^{\mathrm{AB}, \mathrm{a}}$ & $26,27^{\mathrm{B}, \mathrm{a}}$ & 27,02 \\
\hline \multicolumn{5}{|l|}{ Nelore Controle } \\
\hline Nellore Control & $27,20^{\mathrm{A}, \mathrm{a}}$ & $27,88^{\mathrm{A}, \mathrm{a}}$ & $26,64^{\mathrm{A}, \mathrm{a}}$ & 27,24 \\
\hline \multicolumn{5}{|l|}{ Nelore Seleção } \\
\hline Nellore Selection & $29,17^{\mathrm{A}, \mathrm{a}}$ & $26,91^{\mathrm{B}, \mathrm{a}}$ & $26,33^{\mathrm{B}, \mathrm{a}}$ & 27,47 \\
\hline Média (Mean) & 28,13 & 27,08 & 26,70 & \\
\hline
\end{tabular}

Médias, na linha/coluna, seguidas de letras maiúsculas/minúsculas diferentes são diferentes $(\mathrm{P}<0,05)$.

Means, within a row/column, followed by capital/small different letters are different $(P<.05)$.

da maciez de $24-50 \%$, principalmente com carnes com força de cisalhamento $\geq 5,0 \mathrm{~kg}$. Entretanto, os resultados de FC observados (amaciamento de 0,17$0,76 \mathrm{~kg}$ com cloreto de cálcio) são semelhantes aos encontrados por KOOHMARAIE et al. (1990), que obtiveram redução da FC em 1 kg. A utilização de cloreto de cálcio foi importante para reduzir a FC por intermédio do fornecimento de cálcio exógeno. Segundo alguns autores (ALARCON-ROJO e DRANSFIELD, 1995; KOOHMARAIE et al., 1988, 1989, 1990; e KOOHMARAIE E SHACKELFORD, 1991), durante o processo de amaciamento da carne, a quantidade de cálcio liberada pelo retículo sarcoplasmático já é suficiente para ativar m-calpaína (que requer pequena quantidade de íons cálcio para ser ativada), enquanto a m-calpaína permanece intacta (mas disponível para ativação). Assim, a aplicação de cálcio exógeno (na forma de cloreto de cálcio) ativa ambas as calpaínas, resultando em amaciamento maior e mais rápido. Quanto maior a concentração de cloreto de cálcio empregada, menor a FC. Entretanto, com o emprego de maior concentração de cloreto de cálcio (300 mM), observou-se em muitos trabalhos carne com sabor desagradável, caracterizado como um sabor mais salgado, ácido, amargo e metálico, provavelmente devido ao fato de o íon cloreto criar uma sensação metálica, que pode ser contornada com a aplicação de antioxidantes (MILLER et al., 1986; MORGAN et al., 1991; ST. ANGELO, 1991; e BOLES E SHAND, 1993). Quanto ao nível de cloreto de cálcio a ser injetado, segundo WHEELER et al. (1993a), a utilização de $200 \mathrm{mM}$ de cloreto de cálcio foi ideal para eliminar a dureza da carne, sem comprometer outras características de qualidade. Para o efeito da raça dentro da concentração de cloreto de cálcio, o emprego da solução controle fez com que os animais $\mathrm{C}$ apresentassem menor 
1386 Rev. bras. zootec.

FC; os animais NC, valores intermediários aos NS e G; e os G, carne menos macia. Aplicando-se $200 \mathrm{mM}$, os animais $\mathrm{C}$ ainda continuaram apresentando carne mais macia; os G, maior FC; e os NC e NS, valores intermediários de FC. Com a aplicação de 300 mM, a variação na $\mathrm{FC}$ foi reduzida, em que os animais $\mathrm{G}$, $\mathrm{NS}$ e NC apresentaram maior FC e os $\mathrm{C}$, menor FC. Com isso, observa-se a influência do sangue $B o s$ indicus e Bos taurus para estes animais.

Para a interação concentração de cloreto de cálcio x tempo de maturação (Tabela 3 ), observa-se que houve efeito positivo $(\mathrm{P}<0,05)$ do tempo de maturação dentro da concentração, em que houve redução significativa $(0,92-0,94 \mathrm{~kg})$ do $1 \underline{0}$ ao $7 \underline{0}$ dia. No entanto, do 70 para o 14 음 dia, não houve efeito da maturação sobre a FC para as concentrações 200 e $300 \mathrm{mM}$ de cloreto de cálcio $(0,28-0,37 \mathrm{~kg})$, mas somente redução da FC ao 14 o dia com a utilização da solução controle $(0,71 \mathrm{~kg})$. Assim, observa-se que, com a utilização do cloreto de cálcio, o amaciamento da carne foi acelerado (devido ao estado avançado da proteólise), reduzindo-se, portanto, o tempo de maturação que seria necessário para se ter a mesma carne macia. Para muitos autores (KOOHMARAIE et al. 1988, 1990; KOOHMARAIE E SHACKELFORD, 1991; MORGAN et al., 1991; WHEELER et al., 1991; e WHEELER et al., 1992), o tempo de maturação requerido para se obter carne macia é reduzido (para 24 horas) com a aplicação de cloreto de cálcio logo após o abate. Em relação ao efeito da concentração de cloreto de cálcio dentro do tempo de maturação, foi observado que a aplicação de cloreto de cálcio reduziu $(\mathrm{P}<0,05)$ a $\mathrm{FC}$ em todos os tempos de maturação estudados, e para a utilização de 200 ou 300 $\mathrm{mM}$ de cloreto de cálcio essa diferença não foi significativa. $\mathrm{O}$ fornecimento de cálcio exógeno (cloreto de cálcio), portanto, acelera o processo de amaciamento da carne, provavelmente por ativar completamente a $\mu$ - e a m-calpaína, a qual eventualmente resulta em perda de sua atividade, devido à autólise ou, ainda, ao fato de o cálcio induzir mudanças estruturais que quebram a miofibrila, ou mesmo pela perda da atividade da calpastatina, que pode ser hidrolizada pelas calpaínas servindo como um substrato (KOOHMARAIE, 1990 a,b; KOOHMARAIE et al., 1988, 1989, 1990; KOOHMARAIE e SHACKELFORD, 1991; MORGAN et al., 1991; WHIPPLEE KOOHMARAIE, 1992; e WHEELER et al., 1993 a,b).

Perdas por evaporação (PE)

Para a interação raça $\mathrm{x}$ tempo de maturação
(Tabela 1), o tempo de maturação não apresentou influência sobre as raças $\mathrm{C}$ e $\mathrm{NC}(\mathrm{P}<0,05)$, mas para os animais $\mathrm{G}$ e NS as PE foram menores em razão do prolongamento do tempo de maturação. Para o efeito da raça sobre o tempo de maturação, observa-se que, para o dia 1 de maturação, os animais $\mathrm{C}$ apresentaram valores baixos de $\mathrm{PE}$; os NS, os maiores valores de PE; e os G e NC, valores de PE intermediários. Para os períodos de 7 e 14 dias de maturação, as PE não tiveram diferenças estatísticas para todas as raças estudadas. Assim, o tempo de maturação também reduz a variabilidade para as perdas por evaporação. Ainda, animais Bos indicus apresentaram tendência de maiores $\mathrm{PE}$, enquanto animais $B o s$ taurus apresentaram as menores PE. Quanto maior a quantidade de líquidos perdidos nos sete primeiros dias, menores foram as perdas durante o restante do processo de maturação. O empacotamento e processo de maturação podem aumentar e reter, em média, 3,5\% do peso (WHEELER et al., 1993b).

Estudando-se a interação raça x concentração de cloreto de cálcio (Tabela 2), observa-se que não houve efeito da concentração de cloreto de cálcio dentro das raças G, NC e NS, mas, para a raça C, quanto maior a concentração empregada, maiores as perdas por evaporação. Para o efeito da raça dentro da concentração de cloreto de cálcio, a raça não influenciou nas PE.

$\mathrm{Na}$ Tabela 3, são apresentados os resultados da interação entre a concentração de cloreto de cálcio e o tempo de maturação, em que o tempo de maturação não apresentou efeito significativo $(\mathrm{P}<0,05)$ dentro das concentrações estudadas e as concentrações de cloreto de cálcio não tiveram efeito dentro dos tempos de maturação estudados.

Concluiu-se, portanto, que para as interações estudadas as PE para as raças G e NS foram reduzidas com os 14 dias de maturação e, no $1 \underline{0}$ dia de maturação, as raças estudadas apresentaram grande variabilidade nas PE, mas foram reduzidas pela maturação por 7 a 14 dias. A influência da concentração de cloreto de cálcio dentro da raça só foi significativa para a raça $\mathrm{C}$, na qual, aumentando a concentração de cloreto de cálcio, as PE se elevaram. Não houve efeito da interação tempo de maturação $\mathrm{x}$ concentração de cloreto de cálcio e do efeito das raças dentro das concentrações de cloreto de cálcio sobre as PE.

Perdas por gotejamento $(P G)$

Na Tabela 1, observa-se que, para a interação tempo de maturação $\mathrm{x}$ raça, o tempo de maturação 
MOURA et al.

Tabela 2 - Médias da força de cisalhamento, perda por evaporação, perda por gotejamento e perdas totais em relação à raça, concentração de cloreto de cálcio e interação raça x concentração de cloreto de cálcio

Table 2 - Means of the shear force, evaporation loss, drip loss and total loss by breed, calcium chloride concentration and breed vs. calcium chloride concentration interaction

\begin{tabular}{|c|c|c|c|c|}
\hline \multirow[t]{2}{*}{$\begin{array}{l}\text { Raça } \\
\text { Breed }\end{array}$} & \multicolumn{3}{|c|}{$\begin{array}{l}\text { Cloreto de cálcio } \\
\text { Calcium chloride }\end{array}$} & \multirow[t]{2}{*}{$\begin{array}{l}\text { Média } \\
\text { Mean }\end{array}$} \\
\hline & $1 \mathrm{~d}$ & $7 \mathrm{~d}$ & $14 \mathrm{~d}$ & \\
\hline & \multicolumn{3}{|c|}{ Força de cisalhamento $(\mathrm{kg})$ (Shear force) } & \\
\hline Caracu & $3,00^{\mathrm{A}, \mathrm{c}}$ & $2,59^{\mathrm{B}, \mathrm{c}}$ & $2,34^{\mathrm{C}, \mathrm{b}}$ & 2,64 \\
\hline Guzerá & $4,49^{\mathrm{A}, \mathrm{a}}$ & $3,53^{\mathrm{B}, \mathrm{a}}$ & $3,26^{\mathrm{B}, \mathrm{a}}$ & 3,76 \\
\hline Nelore Controle & & & & \\
\hline $\begin{array}{l}\text { Nellore Control } \\
\text { Nelore Seleção }\end{array}$ & $3,95^{\mathrm{A}, \mathrm{ab}}$ & $3,23^{\mathrm{B}, \mathrm{ab}}$ & $3,08^{\mathrm{B}, \mathrm{a}}$ & 3,42 \\
\hline Nellore Selection & $3,90^{\mathrm{A}, \mathrm{b}}$ & $2,98^{\mathrm{B}, \mathrm{bc}}$ & $2,97^{\mathrm{B}, \mathrm{a}}$ & 3,28 \\
\hline \multirow[t]{2}{*}{ Média(Mean) } & 3,84 & 3,08 & 2,91 & \\
\hline & \multicolumn{3}{|c|}{ Perdas por evaporacão (\%) (Evaporation loss) } & \\
\hline Caracu & $14,70^{\mathrm{B}, \mathrm{a}}$ & $15,27 \mathrm{AB}, \mathrm{a}$ & $16,63^{\mathrm{A}, \mathrm{a}}$ & 15,54 \\
\hline Guzerá & $15,05^{\mathrm{A}, \mathrm{a}}$ & $15,92^{\mathrm{A}, \mathrm{a}}$ & $15,84^{\mathrm{A}, \mathrm{a}}$ & 15,60 \\
\hline \multicolumn{5}{|l|}{ Nelore Controle } \\
\hline \multicolumn{4}{|l|}{ Nelore Seleção } & 16,28 \\
\hline Nellore Selection & $16,46^{\mathrm{A}, \mathrm{a}}$ & $17,41^{\mathrm{A}, \mathrm{a}}$ & $16,76^{\mathrm{A}, \mathrm{a}}$ & 16,88 \\
\hline \multirow[t]{2}{*}{ Média(Mean) } & 15,57 & 16,26 & 16,39 & \\
\hline & \multicolumn{3}{|c|}{ Perdas por gotejamento (\%) (Drip loss) } & \\
\hline Caracu & $12,15^{\mathrm{A}, \mathrm{a}}$ & $12,30^{\mathrm{A}, \mathrm{a}}$ & $11,42^{\mathrm{A}, \mathrm{ab}}$ & 11,96 \\
\hline Guzerá & $11,43^{\mathrm{A}, \mathrm{a}}$ & $11,23^{\mathrm{A}, \mathrm{ab}}$ & $11,60^{\mathrm{A}, \mathrm{a}}$ & 11,42 \\
\hline \multicolumn{5}{|l|}{ Nelore Controle } \\
\hline \multicolumn{4}{|l|}{ Nelore Seleção } & 10,96 \\
\hline Nellore Selection & $10,91^{\mathrm{A}, \mathrm{a}}$ & $10,77^{\mathrm{A}, \mathrm{b}}$ & $10,08^{\mathrm{A}, \mathrm{b}}$ & 10,59 \\
\hline Média(Mean) & 11,37 & $\begin{array}{l}11,24 \\
\text { tais }(\%)(T\end{array}$ & 11,09 & \\
\hline Caracu & $26,86^{\mathrm{A}, \mathrm{a}}$ & $27,58^{\mathrm{A}, \mathrm{a}}$ & $28,05^{\mathrm{A}, \mathrm{a}}$ & 27,49 \\
\hline Guzerá & $26,48^{\mathrm{A}, \mathrm{a}}$ & $27,14^{\mathrm{A}, \mathrm{a}}$ & $27,43^{\mathrm{A}, \mathrm{a}}$ & 27,02 \\
\hline Nelore Controle & & & & \\
\hline $\begin{array}{l}\text { Nellore Control } \\
\text { Nelore Seleção }\end{array}$ & $27,06^{\mathrm{A}, \mathrm{a}}$ & $27,08^{\mathrm{A}, \mathrm{a}}$ & $27,59^{\mathrm{A}, \mathrm{a}}$ & 27,24 \\
\hline Nellore Selection & $27,39^{\mathrm{A}, \mathrm{a}}$ & $28,17^{\mathrm{A}, \mathrm{a}}$ & $26,84^{\mathrm{A}, \mathrm{a}}$ & 27,47 \\
\hline Média (Mean) & 26,95 & 27,49 & 27,48 & \\
\hline
\end{tabular}

Médias, na linha/coluna, seguidas de letras maiúsculas/minúsculas diferentes são diferentes $(P<0,05)$.

Means, within a row/column, followed by capital/small different letters are different $(P<.05)$.

dentro da raça não influenciou os animais $\mathrm{G}, \mathrm{NC}$ e NS, entretanto, para os animais C, a maturação por 7 dias reduziu as PG, enquanto a maturação por 14 dias foi igual à maturação por 1 dia. Para o efeito da raça dentro do tempo de maturação, ao 10 dia de maturação, observa-se grande variabilidade nas PG, com maiores perdas para os animais $\mathrm{C}$, seguidos dos animais $\mathrm{G}$ e NC e menores perdas para os NS. Ao 70 dia, esta variabilidade não existiu (foi eliminada), mas, no 14은 dia, novamente se apresentou nas PG. Todavia, os NC tiveram as menores PG, ao invés dos animais NS.

Para a interação raça $\mathrm{x}$ concentração de cloreto de cálcio (Tabela 2), não existiu efeito da concentração de cloreto de cálcio dentro da raça. Entretanto, quando se estudou o efeito da raça dentro da concentração de cloreto de cálcio, constatou-se que, sem cloreto de cálcio (0 mM), as PG são iguais para todas as raças, e para $200 \mathrm{mM}$ as menores PG foram para os animais NC e NS, seguidos dos G, e as maiores PG, para os animais C. Quando da aplicação de $300 \mathrm{mM}$, os animais NS continuaram a apresentar menores $\mathrm{PG}$, seguidos pelos valores intermediários de $\mathrm{PG}$ dos animais $\mathrm{C}$ e $\mathrm{NC}$ e menores $\mathrm{PG}$ dos animais $\mathrm{G}$.

Estudando-se a interação concentração de cloreto de cálcio x tempo de maturação, não se observaram diferenças estatísticas para o efeito do tempo de maturação dentro da concentração de cloreto de cálcio e nem do efeito da concentração de cloreto de cálcio dentro do tempo de maturação (Tabela 3).

\section{Perdas totais (PT)}

Na Tabela 1, verifica-se a influência da interação raça $x$ tempo de maturação sobre as PT, em que o tempo 
1388 Rev. bras. zootec.

de maturação apresentou efeito positivo dentro das raças $\mathrm{G} \mathrm{e} \mathrm{NS}$, que apresentaram menores $\mathrm{PT}$ em virtude do tempo de maturação empregado. Para as raças $\mathrm{C}$ e NC, não foi encontrado efeito para as PT. Para o efeito da raça dentro do tempo de maturação, não foram observadas diferenças para as raças estudadas.

Para a interação raça $\mathrm{x}$ concentração de cloreto de cálcio (Tabela 2), não foram observadas diferenças para os efeitos da concentração de cloreto de cálcio dentro de raça, nem para o efeito da raça dentro da concentração de cloreto de cálcio.

$\mathrm{Na}$ Tabela 3, observam-se diferenças para a interação concentração de cloreto de cálcio x tempo de maturação, constatando-se que, para o efeito do tempo de maturação dentro da concentração, que entre a nãoinjeção de cloreto de cálcio $\left(\begin{array}{lll}0 & \mathrm{mM}\end{array}\right)$ e 300 mM não houve diferenças entre os tempos de maturação estudados. Entretanto, para a concentração $200 \mathrm{mM}$, quanto maior o período de maturação, menores as PT. Para o efeito da concentração de cloreto de cálcio dentro do tempo de maturação, não foram encontradas diferenças estatísticas para esta interação.

Independente do corte cárneo estudado, MORGAN et al. (1991) e WHEELER et al. (1992) não encontraram diferenças entre as perdas por cozimento ou o efeito dos tratamentos empregados.

Tabela 3 - Médias da força de cisalhamento, perda por evaporação, perda por gotejamento e perdas totais em relação à concentração de cloreto de cálcio, ao tempo de maturação e à interação concentração x tempo de maturação

Table 3 - Means of the shear force $(\mathrm{kg})$, evaporation loss, drip loss and total losses on calcium chloride concentration, ageing time and calcium chloride concentration vs. ageing time

\begin{tabular}{|c|c|c|c|c|}
\hline \multirow[t]{2}{*}{$\begin{array}{l}\text { Cloreto de cálcio }(\mathrm{mM}) \\
\text { Calcium chloride }(\mathrm{mM})\end{array}$} & \multicolumn{3}{|c|}{ Maturação } & \multirow[t]{2}{*}{$\begin{array}{l}\text { Média } \\
\text { Mean }\end{array}$} \\
\hline & $1 \mathrm{~d}$ & $7 \mathrm{~d}$ & $14 \mathrm{~d}$ & \\
\hline \multicolumn{5}{|c|}{ Força de cisalhamento (Shear force), $k g$} \\
\hline 0 & $4,70^{\mathrm{A}, \mathrm{a}}$ & $3,76^{\mathrm{B}, \mathrm{a}}$ & $3,05^{\mathrm{C}, \mathrm{a}}$ & 3,84 \\
\hline 200 & $3,82^{\mathrm{A}, \mathrm{b}}$ & $2,85^{\mathrm{B}, \mathrm{b}}$ & $2,57^{\mathrm{B}, \mathrm{b}}$ & 3,08 \\
\hline 300 & $3,65^{\mathrm{A}, \mathrm{b}}$ & $2,73^{\mathrm{B}, \mathrm{b}}$ & $2,36^{\mathrm{B}, \mathrm{b}}$ & 2,91 \\
\hline Média (Mean) & 4,06 & 3,12 & 2,66 & \\
\hline \multicolumn{5}{|c|}{ Perdas por evaporação (Evaporation loss), \% } \\
\hline 0 & $15,90^{\mathrm{A}, \mathrm{a}}$ & $15,73^{\mathrm{A}, \mathrm{a}}$ & $15,09^{\mathrm{A}, \mathrm{a}}$ & 15,57 \\
\hline 200 & $16,84^{\mathrm{A}, \mathrm{a}}$ & $16,82^{\mathrm{A}, \mathrm{a}}$ & $15,11^{\mathrm{A}, \mathrm{a}}$ & 16,26 \\
\hline 300 & $16,91^{\mathrm{A}, \mathrm{a}}$ & $16,61^{\mathrm{A}, \mathrm{a}}$ & $15,64^{\mathrm{A}, \mathrm{a}}$ & 16,39 \\
\hline Média (Mean) & 16,55 & 16,39 & 15,28 & \\
\hline \multicolumn{5}{|c|}{ Perda por gotejamento (Drip loss), \% } \\
\hline 0 & $11,68^{\mathrm{A}, \mathrm{a}}$ & $10,99^{\mathrm{A}, \mathrm{a}}$ & $11,44^{\mathrm{A}, \mathrm{a}}$ & 11,37 \\
\hline 200 & $11,56^{\mathrm{A}, \mathrm{a}}$ & $10,58^{\mathrm{A}, \mathrm{a}}$ & $11,58^{\mathrm{A}, \mathrm{a}}$ & 11,24 \\
\hline 300 & $11,51^{\mathrm{A}, \mathrm{a}}$ & $10,50^{\mathrm{A}, \mathrm{a}}$ & $11,26^{\mathrm{A}, \mathrm{a}}$ & 11,09 \\
\hline Média (Mean) & 11,58 & 10,69 & 11,42 & \\
\hline \multicolumn{5}{|c|}{ Perda total (Total loss), $\%$} \\
\hline 0 & $27,58^{\mathrm{A}, \mathrm{a}}$ & $26,73^{\mathrm{A}, \mathrm{a}}$ & $26,53^{\mathrm{A}, \mathrm{a}}$ & 26,95 \\
\hline 200 & $28,39^{\mathrm{A}, \mathrm{a}}$ & $27,40^{\mathrm{AB}, \mathrm{a}}$ & $26,69^{\mathrm{B}, \mathrm{a}}$ & 27,49 \\
\hline 300 & $28,41^{\mathrm{A}, \mathrm{a}}$ & $27,12^{\mathrm{A}, \mathrm{a}}$ & $26,89^{\mathrm{A}, \mathrm{a}}$ & 27,48 \\
\hline Média (Mean) & 28,13 & 27,08 & 26,70 & \\
\hline
\end{tabular}

Médias, na linha/coluna, seguidas de letras maiúsculas/minúsculas diferentes são diferentes $(P<0,05)$.

Means, within a row/column, followed by capital/small different letters are different $(P<.05)$.

\section{Conclusões}

Os animais zebuínos não apresentaram força de cisalhamento diferente entre si, e sim maior força de cisalhamento quando comparados com os taurinos. Animais Caracu apresentaram menores valores de força de cisalhamento, seguidos pelos animais Nelore Controle e Nelore Seleção, enquanto os animais Guzerá apresentaram maiores valores para a força de cisalhamento, mostrando, portanto, a influência do sangue de animais Bos indicus e Bos taurus sobre a maciez da carne.

Quanto maior o tempo de maturação, menor a força de cisalhamento. Sete dias de maturação foram suficientes para se obter carne macia e com menor variação na maciez da carne injetada com cloreto de cálcio pós-rigor. Entretanto, um dia de maturação também resultou em redução da força de cisalhamento,

A força de cisalhamento foi mais reduzida, quando a concentração de cloreto de cálcio injetada foi 
maior, sendo que $200 \mathrm{mM}$ resultou em redução de $20 \%$ na força de cisalhamento e $300 \mathrm{mM}$ em 24\%, acrescentando, portanto, pouco em relação à primeira. Observa-se que o cloreto de cálcio reduziu a força de cisalhamento, provavelmente pela ativação da mcalpaína, que, em condições pós-morte normais, não é totalmente ativada.

As perdas por evaporação não foram influenciadas pelas raças estudadas, mas, quanto maior o tempo de maturação empregado, menores foram as perdas por evaporação, em que as menores perdas por evaporação foram aquelas obtidas quando as amostras foram maturadas por 14 dias. Quanto maior a concentração de cloreto de cálcio injetada, maiores as perdas por evaporação.

As perdas por gotejamento não foram influenciadas pelas raças, mas a maturação por sete dias reduziu as perdas por gotejamento, enquanto para 1 ou 14 dias foram iguais e maiores que aos 7 dias de maturação. A concentração de cloreto de cálcio não influenciou as perdas por gotejamento,

As perdas totais não foram influenciadas pelas raças e pela concentração de cloreto de cálcio. Já o tempo de maturação influenciou positivamente as perdas totais, em que a maturação por sete dias reduziu as perdas totais.

\section{Referências Bibliográficas}

ALARCÓN-ROJO, A.D., DRANSFIELD, E. 1995. Alteration of post-mortem ageing in beef by the addition of enzyme inhibitors and activators. Meat Sci., 41(2):163-178.

AMERICAN MEAT SCIENCE ASSOCIATION - AMSA. 1978. Guidelines for cookery and sensory evaluation of meat. $24 \mathrm{p}$.

BOLES, J.A., SHAND, P.J. 1993. Effect of calcium injection on the color, retail storage life and sensory properties of beef top round. Recip. Meat Conf. Proc. of Nebraska, 46:71.

CROUSE, J.D., SEIDEMAN, S.C., CUNDIFF, L.V. 1987. The effect of carcass electrical stimulation on meat obtained from Bos indicus and Bos taurus cattle. J. Food Qual., 10(6):407-416.

CROUSE, J.D., CUNDIFF, L.V., KOCH, R.M. et al. 1989. Comparisons of Bos indicus and Bos taurus inheritance for carcass beef characteristics and meat palatability. J. Anim. Sci., 67(10):2661-2668.

FELÍCIO, P.E. A carcaça Nelore para o desossador. In: SEMINÁRIO MANAH “ O NELORE PARA CARNE", 5, Fazenda Mundo Novo, 1995. Anais: Brotas: Fazenda Mundo Novo. p.18-34, 1995.

KOCH, R.M., DIKEMAN, M.E., CROUSE, J.D. 1982. Characterization of biological types of cattle (Cycle III). III. Carcass composition quality and palatability. J. Anim. Sci., 54(1):35-45.

KOOHMARAIE, M., BABIKER, A.S., SCHOROEDER, A.L. et al. 1988. Acceleration of postmortem tenderization process of ovine carcass through activation of $\mathrm{Ca}^{+2}$-dependent proteases. J. Food Sci., 53(6):1638-1641.

KOOHMARAIE, M. CROUSE, J.D., MERSMANN, H.J. 1989.
Acceleration of postmortem tenderization in ovine carcasses through infusion of calcium chloride: effect of concentration and ionic strength. J. Anim. Sci., 67(4):934-942.

KOOHMARAIE, M. 1990a. Quantification of Ca+2-dependent protease activities by hydrophobic and ion-exchange chromatography. J. Anim. Sci., 68(3):659-665.

KOOHMARAIE, M., WHIPPLE, G., CROUSE, J.D. 1990. Acceleration of postmortem tenderization in lamb and Brahmancross beef carcasses through infusion of calcium chloride. J. Anim. Sci., 68(5):1278-1283.

KOOHMARAIE, M. 1990b. Inhibition of postmortem tenderization in ovine carcasses through infusion of zinc. J.Anim. Sci.,68(5):14761483.

KOOHMARAIE, M., SHACKELFORD, S.D. 1991. Effect of calcium chloride infusion on the tenderness of lambs fed $\beta$-adrenergic agonist. J. Anim. Sci., 69(6):2463-2471.

LANSDELL, J.L., MILLER, M.F., WHEELER, T.L. et al. 1995. Postmortem injection of calcium chloride effects of beef quality traits. J. Anim. Sci., 73(6):1735-1740.

MILLER, M.F., DAVIS, G.W., SEIDEMAN, S.C. et al. 1986. Effects of chloride salts on appearance, palatability, and storage traits of flaked and formed beef bullock restructured steaks. $J$. Food Sci., 51(6):1424-1436.

MORGAN, J.B., MILLER, R.K., MENDES, F.M. et al. 1991. Using calcium chloride injection to improve tenderness of beef from mature cows. J. Anim. Sci., 69(11):4469-4476.

SHACKELFORD, S.D., WHEELER, T.L., KOOHMARAIE, M. 1995. Relationship between shear force and trained sensory panel tenderness ratings of 10 major muscles from Bos indicus and Bos taurus cattle. J. Anim. Sci., 73(11):3333-3340.

SAS statistical system user's guide: Stat, Version 6, 4.ed., Cary: SAS Institute, 1990. v.2.

ST. ANGELO, A.J., KOOHMARAIE, M., CRIPPEN, K.L. et al. 1991. Acceleration of tenderization/inhibition of warmed-over flavor by calcium chloride-antioxidant infusion into lamb carcasses. J. Food Sci., 56(2):359-362.

WHEELER, T.L., KOOHMARAIE, M., CROUSE, J.D. 1991. Effects of calcium chloride injection and hot boning on the tenderness of round muscles. J. Anim. Sci., 69(12):4871-4875.

WHEELER, T.L., CROUSE, J.D., KOOHMARAIE, M. 1992. The effect of postmortem time injection and freezing on the effectiveness of calcium chloride for improving beef tenderness. J. Anim. Sci., 70(11):3451-3457.

WHEELER, T.L., KOOHMARAIE, M., LANSDELL, J.L. et al. 1993a. Effects of postmortem injection time, injection level, and concentration of calcium chloride on beef quality traits. J. Anim. Sci. 71(11):2965-2974.

WHEELER, T.L., MILLER, M.F., DALRYMPLE, R.H. 1993b. Calcium injection. Recip. Conf. Proc. of Nebraska, 46:81-83b.

WHEELER, T.L., KOOHMARAIE, M., CROUSE, J.D. 1993c. A calcium chloride injection process to produce guaranteed tender and calcium fortified meat. Beef Research - Progress Report $\mathrm{n}^{\circ}$ 4. U.S. Department of Agriculture, Agricultural Research Service. ARS 71, 187p.

WHIPPLE, G., KOOHMARAIE, M., DIKEMAN, M.E. et al. 1990. Evaluation of attributes that affect longissimus muscle tenderness in Bos taurus and Bos indicus cattle. J.Anim. Sci., 68(9):2716-2728.

WHIPPLE, G., KOOHMARAIE, M. 1992. Freezing and calcium chloride marination effects on beef tenderness and calpastatin activity. J. Anim. Sci., 70(10):3081-3085. 